Check for updates

Cite this: Nanoscale Adv., 2019, 1, 4148

\title{
Reshaped as polyester-based nanoparticles, gallic acid inhibits platelet aggregation, reactive oxygen species production and multi-resistant Gram- positive bacteria with an efficiency never obtained $\dagger$
}

\author{
Silvana Alfei, (D)*a Maria Grazia Signorello, $\ddagger^{a}$ Anna Schito, $\S^{b}$ Silvia Catena $q^{a}$ \\ and Federica Turrini $\Phi^{a}$
}

\begin{abstract}
Natural polyphenols such as Gallic Acid (GA) form an important class of bioactive chemical entities that, having innumerable biological properties, could represent a safer alternative to common drugs against several disorders, including platelet aggregation, radical oxygen species (ROS) hyperproduction, oxidative stress (OS) and bacterial infections. Unfortunately, their clinical uses are limited by pharmacokinetics drawbacks and high sensitivity to environmental factors. In order to overcome these problems and to exploit the GA curative potentials, it has been linked to a biodegradable nanospherical dendrimer matrix, capable of protecting it, thus obtaining a GA-enriched nanosized dendrimer (GAD) endowed with a strong antioxidant capacity. GAD activity as an inhibitor of platelet aggregation and ROS accumulation and its antibacterial efficiency are evaluated here and compared to those of free GA, obtaining outcomes never achieved. Regarding platelet aggregation induced by thrombin and collagen, the GAD proved to be stronger by 7.1 and 7.3 times, respectively. Furthermore, the GAD showed a ROS inhibitory activity higher than that of GA by 8.1 (thrombin) and 6.9 (collagen) times. Concerning the antibacterial activities, evaluated on eleven multi-resistant Gram-positive strains of clinical relevance, the GAD is far more potent than GA, by exerting a growth inhibitory activity at MIC $(\mu M)$ concentrations lower by factors in the range $12-50$.
\end{abstract}

Received 16th July 2019

Accepted 12th September 2019

DOI: 10.1039/c9na00441f

rsc.li/nanoscale-advances

\section{Introduction}

Oxidative stress (OS)

Chemically reactive molecules containing oxygen or nitrogen atoms are termed reactive oxygen species (ROS) or reactive nitrogen species (RNS), respectively. A variety of sources, both endogenous and exogenous, exposed to different physicochemical conditions or pathophysiological states, can produce ROS and RNS.

$\mathrm{NO}^{\circ}$ is the most reactive among RNS and is produced from the metabolism of $\mathrm{L}$-arginine by the action of the enzymes nitric oxide synthases (NOSs). NOSs catalyze the conversion of $\mathrm{L}^{-}$

\footnotetext{
${ }^{a}$ Department of Pharmacy (DiFAR), University of Genoa, Viale Cembrano 4, I-16148 Genova, Italy.E-mail: alfei@difar.unige.it

${ }^{b}$ Department of Surgical Sciences and Integrated Diagnostics (DISC), University of Genoa, Viale Benedetto XV, 6, I-16132 Genova, Italy

$\dagger$ Electronic supplementary information (ESI) available. See DOI: 10.1039/c9na00441f

\$ This co-author contributed to the evaluation of ROS and platelet aggregation inhibition.

$\S$ This co-author contributed to the evaluation of antibacterial activity.

I These co-authors contributed to the evaluation of RSA\% activity.
}

arginine into L-citrulline and $\mathrm{NO}^{*}$ by a 5 -electron oxidation of a guanidine nitrogen of $\mathrm{L}$-arginine. ${ }^{2}$

ROS have roles in normal cell signaling and homeostasis ${ }^{1,3}$ and cells have a variety of defense mechanisms that intercept free radicals to prevent or limit intracellular damage.

Some low molecular-weight cellular antioxidants, such as ascorbic acid, vitamin $\mathrm{E}$ and glutathione, and antioxidant enzymes, such as thioredoxins, superoxide dismutase (SOD), catalase and glutathione peroxidase, work to keep the dangerous activity of ROS under control. ${ }^{1,3,4}$ However, with increased levels of ROS, defense systems can be overwhelmed, resulting in cellular damage. Normally functioning cells can sustain and tolerate background levels of damage, but if an imbalance and overproduction occur, OS becomes uncontrollable. OS induce significant modification of intracellular targets such as DNA, proteins and lipids and may modulate survivalsignaling cascades, causing increasing cellular damage. Nowadays, OS is considered the key triggering factor for the onset and development of several disorders, including cardiovascular malfunction, cataracts, cancers, rheumatism and many other auto-immune and neurodegenerative diseases and ageing. ${ }^{5} \mathrm{~A}$ number of synthetic drugs may provide protection against the 
deleterious effects of OS but these are also associated with adverse side effects. ${ }^{5}$

\section{Activated platelet aggregation}

Activated platelets have been demonstrated in a wide variety of clinical settings including peripheral arterial disease and also play a key role in the development of acute coronary syndrome and contribute to cerebrovascular events. Platelet activation triggered by inflammation is a critical component of atherothrombosis. ${ }^{6}$ In addition, platelets participate in the process of forming and extending atherosclerotic plaques. ${ }^{7}$ In a large-scale prospective human study, the risk of future cardiovascular events increased with increasing levels of plasma platelet leukocyte aggregation. ${ }^{8}$

Following injury to blood vessels, collagen undergoes activation through a tyrosine kinase-dependent mechanism leading to the release of several biologically active substances and aggregates. Collagen is a vessel wall protein that directly activates platelets. ${ }^{9}$ Platelet activation by exposed collagen after vessel injury is believed to be an early step in cardio-pathogenic disease. ${ }^{10}$

In the following phase of the recruitment of non-adherent platelets, locally generated thrombin, secreted ADP and released TXA2 are greatly involved. Platelet response to thrombin includes reorganization of the actin cytoskeleton, secretion of the content of platelet storage granules, exposure of a fibrinogen receptor on the integrin $\alpha_{\mathrm{II}} \beta 3$ and platelet aggregation. ${ }^{11}$

\section{Bacterial infections}

Antibiotics have been considered as the preferred drugs for curing infections since their discovery. However, during the past few decades, the rapid increase of such infective conditions, associated with a wide and massive use of these therapeutic drugs, has led to the emergence of several antibioticresistant strains, especially in Gram-positive pathogens such as methicillin-resistant Staphylococcus aureus (MRSA) and $S$. epidermidis (MRSE) or vancomycin resistant Enterococcus faecium and E. faecalis (VRE). ${ }^{12-14}$

$S$. aureus and MRSA are major pathogens for humans, causing hospital and community-acquired infections, such as sepsis, pneumonia, skin and soft tissue infections, endocarditis, and many other diseases. ${ }^{15,16}$

MRSE, due to its capability to produce biofilms, can cause life threatening infections, like those on prostheses and intravenous catheters or even endocarditis, in carriers of valvular lesions. ${ }^{17}$

Enterococcus faecium and Enterococcus faecalis are characterized by high levels of intrinsic and acquired antibiotic resistance, particularly to beta-lactam antibiotics and glycopeptides such as vancomycin (VRE).$^{13}$ They are involved in several serious conditions, causing urinary tract infections, bacteremia, endocarditis and meningitis. ${ }^{13}$

Micrococcus luteus is a Gram-positive human commensal that may occasionally cause invasive diseases, usually in immunocompromised patients, such as pneumonia, meningitis associated with ventricular shunts, septic arthritis, bacteremia, peritonitis, endophthalmitis and endocarditis. ${ }^{18}$

Only very few drugs are available at present against MRSA, MRSE and VRE and resistance is still increasing. Therefore the number of effective molecules is dramatically diminishing. ${ }^{19,20}$

Consequently, there is an urgent need for exploring new drugs with high efficacy and low toxicity as alternatives to antibiotics. In this context, naturally occurring antimicrobial agents represent an interesting choice with reduced potential impact on the environment and health.

\section{Natural polyphenols: gallic acid (GA)}

Polyphenols, flavonoids and carotenoids are phytochemicals present in unprocessed or minimally processed plant foods such as fruits, vegetables, grains, beans, legumes, herbs, spices, nuts and seeds. Such compounds form an important class of bioactive entities, having innumerable biological activities such as anticancer, antifungal, antibacterial, antiviral, antiulcer and anti-cholesterol activities, all attributable to a remarkable antioxidant power. They represent a naturally occurring alternative to common drugs against OS.

The screening of safe and effective anti-tumor compounds from Traditional Chinese Medicine is currently a hotspot of research, and a number of effective components isolated from plants have been revealed to possess significant activities and cytotoxic effects against certain cancer cells. ${ }^{21}$

Epidemiological studies have suggested that red wine consumption is related to a reduction in overall mortality caused by cardiovascular events induced by atherothrombosis. ${ }^{22}$

The exact nature of the protective effect of red wine is still unclear, but it probably depends on the capability of wine constituents to reduce the progression of atherosclerotic lesions. ${ }^{23}$ Protective effects against cardiovascular diseases have also been reported for green tea. ${ }^{24}$

Furthermore, natural products such as propolis extracts (which contain phenolic acids, flavonoids, terpenes, and essential oils) have been proved to be effective in the control of some infectious diseases caused by bacteria, even those of the sporogenic type.

As a consequence, recently, growing interest has been focused on using "natural" bioactive compounds from plants as safer and more efficient substitutes for conventional drugs including antibiotics. ${ }^{25-27}$ Concerning bacterial contaminations, phytochemicals are potentially useable in both humans and animals to fight resistant infections as well as in foods or food packaging as preservatives. ${ }^{28}$

3,4,5-Trihydroxybenzoic acid, also named gallic acid (GA), is a naturally occurring polyphenolic phytochemical contained in a wide range of herbal plants, green tea, grapes, strawberries, pineapples and bananas. ${ }^{29,30} \mathrm{~A}$ common and normal feeding regime leads to the daily intake of GA which is reasonably regarded as "natural" and "safe" for humans. It is a low molecular weight compound with a simple chemical structure (Fig. 1), but food chemists consider it a multi-target nutraceutical, i.e. a "pharmaceutical-grade nutrient". 


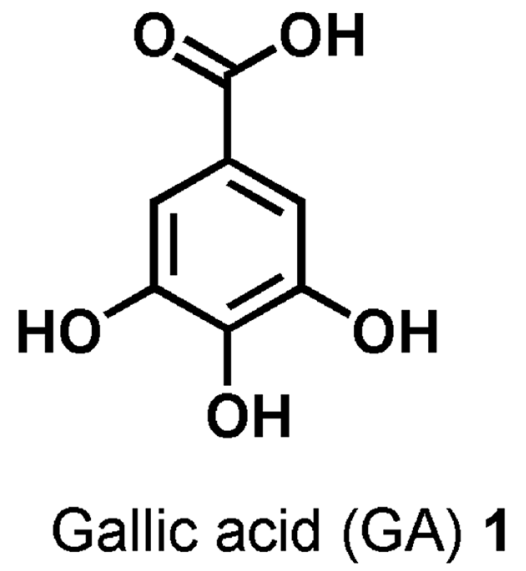

Fig. 1 Structure of gallic acid (GA) 1.

In addition to its nutritional values, it has a natural and marked antioxidant power and has been shown to be effective against OS. ${ }^{5,31-33}$

GA, by inhibiting the overproduction of free radicals and by limiting OS, which are the main causes of the onset and development of most diseases, is effective against most of them. It shows antiallergenic, anti-mutagenic, anti-carcinogenic, antiviral, antibacterial and anti-inflammatory activities. ${ }^{34-37}$ Furthermore, it shows anti-neurodegenerative and regenerative properties, as well as anti-atherosclerosis effects.

GA is able to exert remarkable antioxidant activity also in emulsion or lipid systems, ${ }^{32,33}$ and consequently, is used in processed foods, cosmetics and food packing materials to prevent rancidity induced by lipid peroxidation and spoilage. ${ }^{28}$

GA has been identified as an active component of certain herbal medicinal plants, which exhibit growth inhibitory effects on various cancer cell lines. ${ }^{38-40}$

In addition, GA plays a significant role in the prevention of malignant transformation and development of cancer in vivo. ${ }^{\mathbf{1 1}}$

Besides a considerable anti-mutagenic and anti-carcinogenic activity, ${ }^{\mathbf{4 2 - 4 4}}$ GA showed neuroprotective activity in different animal models of OS, under conditions of neurodegeneration and neurotoxicity triggered by OS such as Parkinson's disease. ${ }^{45}$

GA shows antithrombotic and anti-atherosclerotic properties through non-antioxidant mechanisms. GA exerts this latter activity by concentration-dependent inhibition of platelet aggregation, P-selectin expression, and platelet-leukocyte aggregation.

It prevents the elevation of intracellular calcium and attenuated phosphorylation of PKC $\alpha /$ p38 MAPK and Akt/GSK3 $\beta$ on platelets stimulated by ADP or U46619. ${ }^{46}$

Previous studies on GA antibacterial activity have shown very high MIC values against a wide spectrum of bacterial species such as Escherichia coli, ${ }^{\mathbf{4 7}}$ thermophilic Campylobacter, ${ }^{\mathbf{4 8}}$ Staphylococcus aureus, ${ }^{49}$ and Pseudomonas strains ${ }^{50}$ and also against sporogenic Paenibacillus larvae. ${ }^{51}$

Considering all these properties, GA appears to be a promising candidate to be used as such, or as a template molecule, for the design and development of innovative drugs aimed at treating diseases where common therapies are always less effective or are associated with serious collateral events. Unfortunately, the clinical application of GA is limited by its pharmacokinetic drawbacks, such as poor oral bioavailability, diminished absorption in the gastrointestinal tract (GIT), fast metabolism and rapid urine excretion. ${ }^{52-55}$ In addition, like analog polyphenols from plants, GA is also very sensitive to environmental factors such as light, heat, and oxygen. It is also susceptible to autoxidation in aqueous solution in the presence of oxygen.

In order to increase GA stability without losing, but improving, its bioactivity, various approaches, including its association with or inclusion in nanostructured matrices, have been explored.

GA has been bonded to organic polymers such as gelatin and chitosan, entrapped in cyclodextrins, and linked to cheap and inert inorganic materials such as silica nanoparticles. ${ }^{56-61}$

By making use of nanoparticles (NPs), the solubility, the GIT absorption and the metabolic profile of GA, and in general of all bioactive chemical entities, can be ameliorated simultaneously. ${ }^{62}$

Among nanoparticles, dendrimers are endowed with several nonpareil properties, and thus they are extraordinarily attractive and extensively exploited as ideal carriers for both gene and drug delivery. In brief, dendrimers are highly branched and symmetric macromolecules characterized by a monodisperse tree-like structure with both internal cavities for guest molecule entrapment and many peripheral groups that allow further functionalization. Compared to traditional polymers, they have an unusually low intrinsic viscosity that allows their easy transport in the blood. ${ }^{63-67}$ Within the wide category of dendrimers, polyester-based dendrimer scaffolds are the most attractive for biomedical applications because of their good biodegradability, ${ }^{68-70}$ which the well known poly(amidoamine) dendrimers (PAMAMs) don't possess. ${ }^{71}$ Based on all these considerations, recently, a fifth generation nanosized polyesterbased dendrimer [named $\mathbf{4}$ in the ESI, Scheme S1 and Section $\mathrm{S} 1 \dagger]$ was prepared and peripherally decorated with 64 GA units, achieving a GA-enriched nanospherical dendrimer GAD (named 9 in the ESI, Scheme S1 and Section 1†) (Fig. 2). ${ }^{72}$

The GAD showed no cytotoxicity ${ }^{72}$ (Section S4.2 in the ESI $\dagger$ ) and therefore was not harmful to cells, and it exhibited a radical scavenging activity four times higher than that of free GA (Section S5 in the ESI $\dagger$ ). The GAD proved to be totally biodegradable and able to release bioactive GA units by cell esterase attack for further antioxidant activity.

A study concerning the efficiency of the GAD as a preservative additive to prevent essential oil (EO) auto-oxidative spoilage confirmed its high performance as a radical scavenger and antioxidant. ${ }^{73}$

It was shown to be a preservative additive far more potent than GA, with the ability to stop oxidative degradation thermally induced at very early stages thus improving the EO shelf life (Section S6 in the ESI $\dagger$ ). Furthermore, different from GA, the GAD showed no pro-oxidant activity, even for prolonged exposure to heat, light and air. ${ }^{73}$ 


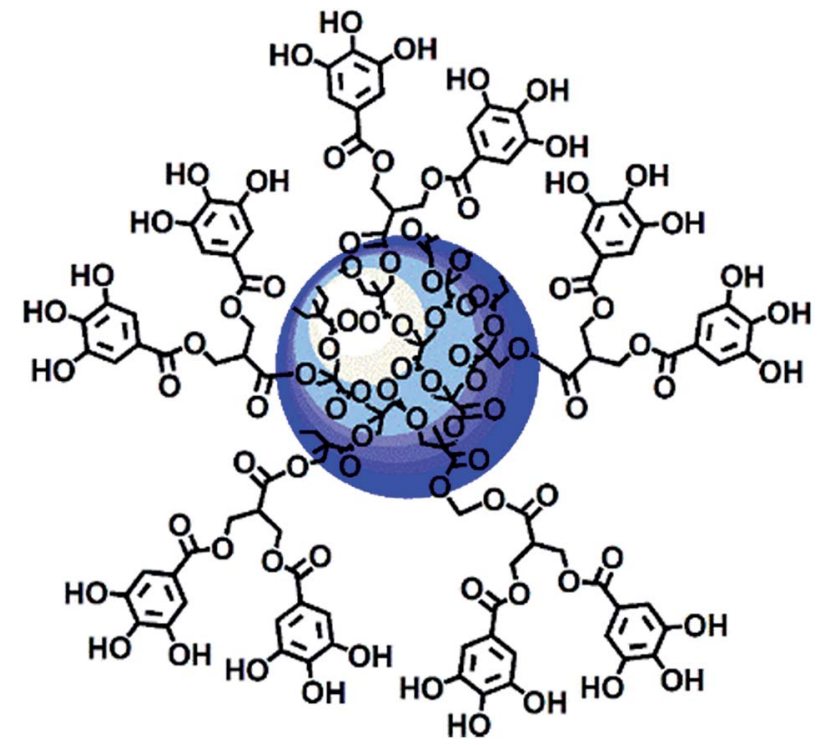

Fig. 2 Intuitive representation of the GAD structure. ${ }^{72}$

In this work, the GAD was proved to also have pharmacological activities at levels never achieved and to be eligible as a potential innovative semi-natural and safe therapeutic nanodevice, more active than GA or than other GA formulations previously prepared.

The GAD was first investigated to evaluate its activity in inhibiting platelet aggregation and intra-platelet ROS hyperproduction which causes OS which in turn induces a cascade of damages that can promote the onset of many diseases. Then, the GAD was also evaluated as an antimicrobial agent against a set of eleven multidrug-resistant bacterial strains of clinical interest. For direct comparison purposes, the antithrombotic and antimicrobial activities of GA were also assayed.

\section{Results and discussion}

In order to ameliorate GA pharmacokinetic drawbacks, to improve its GIT absorption, metabolic profile and stability, a GA-enriched nanospherical dendrimer (GAD) of the fifth generation was recently prepared (Fig. 2 and 3). ${ }^{72}$ Its design was based on the consideration that nanoparticles and dendrimers

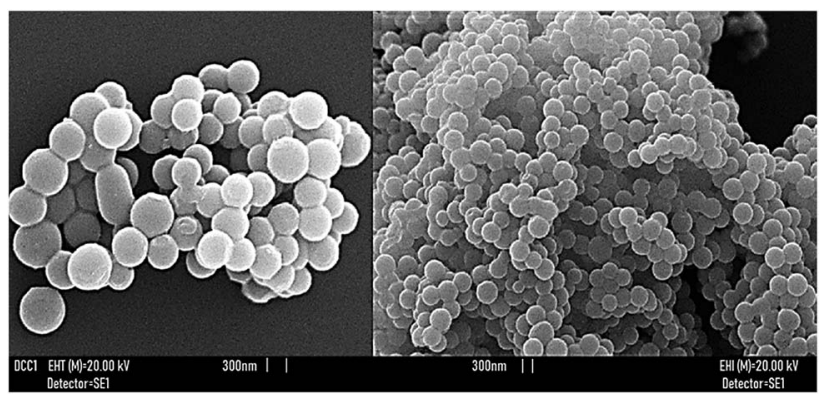

Fig. 3 SEM images of GAD particles. ${ }^{72}$ can enhance the Hydrophilic Lipophilic Balance (HLB), the oral and GIT absorption of drugs, and their blood residence time.

\section{Improvement of drug absorption}

NPs, including dendrimers, may act as an "excipient" or "permeability enhancer", thus altering the barrier function of the intestinal epithelium and thereby enhancing the permeability of a co-administered drug. ${ }^{74}$

If carefully designed, NPs and dendrimer-drug complexes commonly provide improved HLB, which contributes to better dissolution in GIT fluids and to easier transport across the intestinal epithelium. ${ }^{74}$

In the present case, the fifth generation polyester-based dendrimer scaffold adopted as a carrier for GA, would ensure a reduction in the HLB value of GA, providing a GA-reservoir compound with an improved HLB, a more hydrophobic character and a better absorption in the GIT. ${ }^{74,75}$

\section{Improvement of blood residence time}

Large constructs of drug complexes, endowed with high molecular weights and high surface area, are typically retained in the circulation for longer periods and are metabolized more slowly. Concerning transported drugs, higher systemic residence times translate to an enhanced opportunity for acting and to improved therapeutic effects. ${ }^{74}$ High-generation dendrimers possess considerably high molecular weights and the GAD has a significantly high molecular weight estimated $\left({ }^{1} \mathrm{H}\right.$ NMR) to be around 17 000. In addition, the amazing spherical morphology of its particles (Fig. 3), as shown by SEM analysis ${ }^{72}$ further contributes to the desired high surface.

As shown in Fig. 3, according to the scale bar, the GAD particle size was about $300 \mathrm{~nm}$; this value was also validated by GAD particle hydrodynamic size (DLS) analysis which provided a ZAVE size $(\mathrm{nm})$ of $348.6 \pm 2.8$ at $25{ }^{\circ} \mathrm{C}$ (Section S4.2 in the ESI $\dagger$ ).

As we all know, a particle size of about $300 \mathrm{~nm}$ is uncommon for dendrimers of similar generation ${ }^{76}$ but is typical for so called megamers. ${ }^{77}$ Megamers are dendrimer multi-molecular assemblies that, besides occurring thanks to cross-linking agents introduced during their synthesis, can form because of the natural clustering assemblies of dendrimer molecules into supramolecular assemblies. In the present case, such an assembly process was rationally favoured by the several poly hydroxylated gallic acid units, that can establish many hydrogen bonds between the dendrimer molecules, giving rise to dendrimer aggregates.

GAD activity against OS caused by platelet aggregation and intra-platelet ROS production

The effect on human platelets of the GAD was tested in vitro by measuring aggregation and inhibition of ROS production induced through stimulation by either collagen or thrombin.

\section{Collagen}

Collagen is the most thrombogenic component of the sub endothelial layer following vascular injury. Collagen supports 
platelet adhesion to the sub endothelium and induces aggregation, secretion and pro-coagulant activity. The interaction between collagen and platelets is mainly mediated by two receptors, integrin $\alpha 2 \beta 1$ and glycoprotein VI. Glycoprotein VI plays a central role in the signaling pathway leading to the formation of calcium and NO which regulate platelet function and stimulate activation of p72syk and PLC $\gamma 2$ (ref. 78) with subsequent formation of diacylglycerol and activation of protein kinase $\mathrm{C}$ (PKC) and inositol 1,4,5-trisphosphate and calcium release from the dense tubular system. In addition, collagen stimulates platelet calcium influx. ${ }^{79}$ The collagen effect is dose and time-dependent.

\section{Thrombin}

Thrombin is the strongest platelet-aggregating agent in vivo and the protease-activated receptors (PAR) 1 and 4 play a key role in thrombin-induced aggregation. ${ }^{80}$

By binding to heptahelical receptors coupled to heterotrimeric $G$ proteins and through a path other than that of collagen involving PLC $\beta$ thrombin the production of the same second messengers is induced, i.e. diacylglycerol and inositol 1,4,5-trisphosphate which stimulate PKC and calcium elevation, respectively.

\section{Collagen and thrombin-induced platelet aggregation}

The inhibition of platelet aggregation induced both by collagen and thrombin by different GAD and GA concentrations was evaluated after 6 minutes as dictated by the common protocol and the results are shown in Fig. 4.

For a proper interpretation of Fig. 4 and subsequently of Fig. 5, it should be noted that the $\mu \mathrm{M}$ concentration of GA used is higher by 10 times than that of the GAD.

The inhibition of platelet aggregation (IPA\%) and the ROS count derivable from Fig. 4 and 5 for GA and the GAD must be weighted and considered accordingly.

For more clarity, Table 1 summarizes the relative $\mathrm{IC}_{50}$, i.e. the molar concentration of the agent necessary to give $50 \%$ inhibition of the maximal aggregation induced by the agonist used

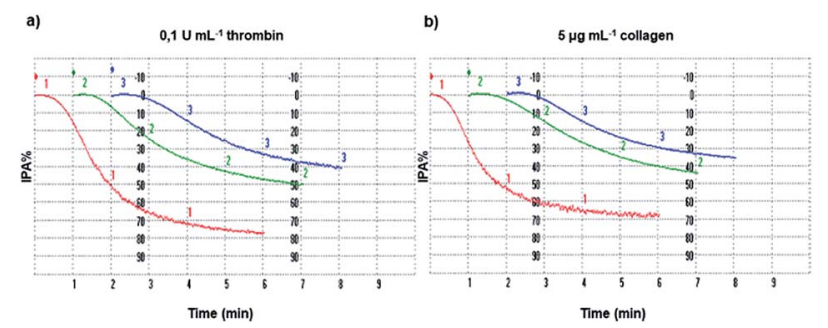

Fig. 4 Platelet aggregation. Washed platelets $\left(3.0 \times 10^{8}\right.$ platelets $\mathrm{mL}^{-1}$ ), pre-incubated at $37^{\circ} \mathrm{C}$ in the presence of saline (line 1 , red), 10 $\mu \mathrm{M}$ GAD (line 2, green) or $100 \mu \mathrm{M}$ GA (line 3, blue), were stimulated with thrombin (a) or collagen (b) as indicated. Platelet aggregation was monitored as described in the Methods section and quantified by the light transmission reaching within 6 min. Tracings are representative of at least six independent experiments.

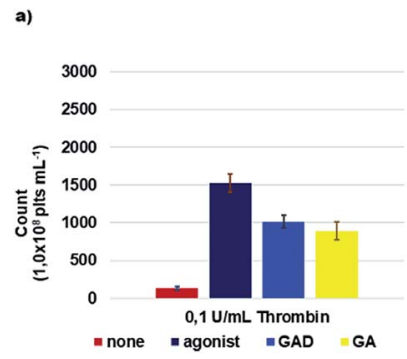

Fig. 5 ROS formation. Washed platelets $\left(1.0 \times 10^{8}\right.$ platelets $\left.\mathrm{mL}^{-1}\right)$, loaded with $10 \mu \mathrm{M}$ DCFH-DA, were pre-incubated with saline, $10 \mu \mathrm{M}$ GAD or $100 \mu \mathrm{M}$ GA and then stimulated for 15 min with thrombin (a) or collagen (b) as indicated. At the end of incubation, samples were immediately analyzed by flow cytometry. Data are the mean \pm SD of at least six experiments carried out in duplicate.

as the stimulant. An additional histogram of the $\mathrm{IC}_{50}(\mu \mathrm{M})$ of the GAD and GA is available in the ESI (Section 7 and Fig. S7.1†).

The GAD inhibited platelet aggregation induced by the two agonists with similar $\mathrm{IC}_{50}$ values of $14.8 \mu \mathrm{M}$ (thrombin) and 14.9 $\mu \mathrm{M}$ (collagen). Comparing the potency of the GAD with that of free GA tested under the same conditions, it proved to be stronger by 7.1 times when aggregation was stimulated by thrombin and by 7.3 times when collagen was used as the agonist.

In addition, according to some literature data ${ }^{\mathbf{8 1 - 8 3}}$ concerning the inhibitory activity of platelet aggregation of aspirin ${ }^{81}$ and of other naturally occurring polyphenols such as 2-(3,4-dihydroxyphenyl)-ethanol (DHPE) in olive oil, ${ }^{81}$ tumerone, ${ }^{82}$ curcumin $^{82}$ and protocatechuic acid (PA) ${ }^{83}$ from several vegetables, the results obtained for the GAD showed a stronger activity. Briefly, when collagen was the agonist, GAD activity results were higher by 2.6, 4.5 and more than 13 times those of aspirin, DHPE and PA, respectively. When activation was induced by thrombin, the activity of the GAD was higher by more than 13 times that of all compounds reported.

\section{Inhibition of agonist-induced ROS production}

As reported, ROS are generated within activated platelets and play an important role in regulating platelet responses to collagen and thrombin and to collagen and thrombin-mediated thrombus formation. ${ }^{\mathbf{8 4 , 8 5}}$ GAD and GA efficiency in inhibiting ROS production within activated platelets by $0.1 \mathrm{U} \mathrm{mL}^{-1}$ thrombin or by $5 \mu \mathrm{g} \mathrm{mL} \mathrm{m}^{-1}$ collagen was investigated. The GAD and GA inhibited ROS production in a concentration-dependent manner (Fig. 5).

Table 1 Platelet aggregation inhibitory activity of the GAD compared to that of $\mathrm{GA}$ expressed as $\mathrm{IC}_{50}(\mu \mathrm{M})$

\begin{tabular}{lll}
\hline Compound & $\begin{array}{l}\mathrm{IC}_{50}(\mu \mathrm{M}) \\
\text { thrombin-induced } \\
\left(0.1 \mathrm{U} \mathrm{mL}^{-1}\right)\end{array}$ & $\begin{array}{l}\mathrm{IC}_{50}(\mu \mathrm{M}) \\
\text { collagen induced } \\
\left(5 \mu \mathrm{mL}^{-1}\right)\end{array}$ \\
\hline GAD & $14.8 \pm 1.5$ & $14.9 \pm 0.5$ \\
Gallic acid (GA) & $105.5 \pm 8.4$ & $109.5 \pm 6.9$
\end{tabular}


Table 2 reports the obtained results expressed as $\mathrm{IC}_{50} \mu \mathrm{M}$, while an additional histogram of $\mathrm{IC}_{50}(\mu \mathrm{M})$ of the GAD and GA is available in the ESI (Section S7 and Fig. S7.2†).

The GAD was a better radical scavenger compared to GA assayed under the same conditions, showing a ROS inhibitory activity higher by 8.1 times when thrombin was used as the inducing agent and by 6.9 times when collagen was used.

It should be noted that the GAD radical inhibitory activity is higher in this investigation performed on cells (platelets) than in the DPPH test. ${ }^{72}$ A rational explanation could be as follows.

As previously reported, the GAD is endowed both with an intrinsic Radical Scavenging Activity (RSA\%) four times higher than that of GA (DPPH test) and with the ability to release the transported GA antioxidant units once inside cells by the cells' esterase hydrolytic activity. ${ }^{72}$

This further discharge of active GA units, which can only occur in cells, suggests that the results of the DPPH test may be an underestimate of the actual RSA power of the GAD. Results reported in Table 2 provide a confirmation of this early assumption. In activated platelets, the GAD demonstrated a ROS inhibitory activity 7-8 times higher than that of GA and therefore 3-4 times higher than that in the DPPH test.

In light of these results, the GAD represents a novel noncytotoxic, semi-natural, nano-technological device, far more potent than free GA, other natural polyphenols or existing therapeutics such as aspirin in regulating platelet aggregation, ROS production and OS and in treating related diseases.

\section{Antibacterial activity of the GAD and GA}

Antibacterial activity of free GA and the GAD was evaluated on different bacterial strains belonging to Gram-positive and -negative species. Minimal Inhibitory Concentration (MIC) values were obtained by following the microdilution method, according to the Clinical and Laboratory Standards Institute protocols. $^{86}$

MIC values of the GAD and GA, expressed in $\mu \mathrm{M}$, are reported in Table 3. GA and the GAD were both totally ineffective against the three Gram-negative species tested (Escherichia coli, Pseudomonas aeruginosa and Klebsiella pneumoniae), showing MIC values in excess of 1506 and $120 \mu \mathrm{M}$, respectively (data not shown).

These findings agree with the high MIC $(\mu \mathrm{M})$ values reported for GA and GA-loaded nanoparticles (GA-NPs) in the recent literature, against $E$. $\operatorname{coli}^{47,76}$ and several other different species of Gram-negative pathogens such as Pseudomonas, Plesiomonas, Salmonella typhimurium and Shigelloides. ${ }^{50,87,88}$

Table 2 ROS inhibitory activity of the GAD compared to that of GA expressed as $I C_{50}(\mu M)$

\begin{tabular}{lcl}
\hline & $\begin{array}{l}\mathrm{IC}_{50}(\mu \mathrm{M}) \\
\text { thrombin-induced } \\
\left(0.1 \mathrm{U} \mathrm{mL}^{-1}\right)\end{array}$ & $\begin{array}{l}\mathrm{IC}_{50}(\mu \mathrm{M}) \\
\text { collagen induced } \\
\left(5 \mu \mathrm{gL}^{-1}\right)\end{array}$ \\
\hline GAD & $14.9 \pm 1.1$ & $12.1 \pm 1.1$ \\
Gallic acid (GA) & $120.4 \pm 10.2$ & $83.8 \pm 7.9$
\end{tabular}

Table 3 Minimal inhibitory concentrations (MICs) ( $\mu M$ ) of the GAD and GA against selected Gram-positive pathogens ${ }^{a}$

\begin{tabular}{lll}
\hline Strains & $\mathrm{GAD}(\mu \mathrm{M})$ & $\mathrm{GA}(\mu \mathrm{M})$ \\
\hline S. aureus 18 (MRSA) & 30 & 377 \\
S. aureus 6 (MSSA) & 15 & 753 \\
S. aureus ATCC29813 & 30 & 753 \\
S. epidermidis 22 (MRSE) & 15 & 377 \\
S. epidermidis 6 (MSSE) & 15 & 377 \\
S. epidermidis ATCC35984 & 30 & 753 \\
E. faecalis 1 (VRE) & 60 & 1506 \\
E. faecalis ATCC29212 & 60 & 753 \\
E. faecium 152 (VRE) & 30 & 753 \\
E. faecium 2 (VSE) & 30 & 1506 \\
M. luteus & 30 & 377 \\
\end{tabular}

${ }^{a}$ MRSA: methicillin-resistant $S$. aureus, MSSA: methicillin-susceptible $S$. aureus, MRSE: methicillin-resistant $S$. epidermidis, MSSE: methicillinsusceptible $S$. epidermidis, VRE: vancomycin-resistant Enterococcus, VSE: vancomycin-susceptible Enterococcus.

These results are probably due to the particular structure of the external layers of these pathogens that allow Gram-negative bacteria to be more refractory to many natural compounds than Gram-positive species.

Regarding the Gram-positive bacteria, our data show that both the GAD and GA behave as powerful inhibitors of the growth of several species of clinical relevance, such $S$. aureus, $S$. epidermidis, E. faecalis, and E. faecium. Interestingly, the two molecules were active on both susceptible and multi-resistant strains of the same species such as MRSA, MRSE and VRE. Moreover, it is worth emphasizing that the GAD is much more active, in terms of MIC values, than the free GA, showing an inhibitory activity at concentrations $(\mu \mathrm{M})$ that were lower than those shown by GA by 12 to 50 times (Table 3 ).

In order to compare the antimicrobial activity of the GAD with that of GA previously reported, careful literature research was performed. Interestingly, while many studies were on the antimicrobial activity of phenolic extracts from several medicinal plants towards Gram-positive strains, data about pure GA are very rare. In addition, when the literature data are available, they are often discordant and differ from the data obtained in this study.

No research exists on the activity of pure GA against $M$. luteus but only a small number of studies reported the inhibitory activity of phenolic extracts on this species with a known content of GA as in the case of Zare et $a l^{89}$

Regarding $S$. aureus, Borges et al. ${ }^{90}$ showed an MIC value of $10294 \mu \mathrm{M}$, while in contrast, Salha and Al-Zahrani ${ }^{91}$ showed MIC values in the concentration range from 20.6 to $73.5 \mu \mathrm{M}$ against MRSA (21-74 $\mu \mathrm{M})$ and MSSA (37-74 $\mu \mathrm{M})$. Fu et al. ${ }^{92}$ reported MIC values of $3706 \mu \mathrm{M}$ against $S$. aureus CMCC(B) 26003 and of $17706 \mu \mathrm{M}$ against MRSA while Alves et al. ${ }^{93}$ found MIC values $>5882 \mu \mathrm{M}$ against either MRSA or MSSA. However, also when considering the lowest reported MIC values that are in the range 21-74 $\mu \mathrm{M}$, the GAD was more efficient than GA by 1.4-2.5 times.

Similarly, the studies on GA activity against $S$. epidermidis are very scarce. Fu et al.,$^{\mathbf{9 2}}$ demonstrated for this compound an MIC 
value of $3706 \mu \mathrm{M}$ against this pathogen while Alves et al.,$^{93}$ reported an MIC $>5882 \mu \mathrm{M}$. Against this species, the GAD was thus effective at lower concentrations by factors in the range 124-247 and 186-392, respectively.

Regarding the Enterococcus genus, Alves et al., ${ }^{93}$ showed MIC values $>5882 \mu \mathrm{M}$ against $E$. faecalis and Gutiérrez-Fernández et $a l .{ }^{94}$ reported MIC values in the range from 17000 to 19400 $\mu \mathrm{M}$, also against VRE, confirming that the GAD is more active than GA by a factor $>98$ according to the results reported by Alves and by factors in the range 283-323 compared to the results reported by Gutiérrez-Fernández.

On the contrary, Kuete ${ }^{95}$ reported for GA an MIC value of 188 $\mu \mathrm{M}$ against VRE E. faecium. Also in this case the GAD was 6.3 times more powerful than GA.

According to our results, the GAD could represent an innovative semi-synthetic non-cytotoxic nanosized therapeutic agent to be considered in order to counteract clinical conditions sustained by drug-refractory Gram-positive pathogens.

\section{Experimental}

\section{Materials and methods}

All the reagents including gallic acid and solvents were purchased from Merck (formerly Sigma-Aldrich). Reagents were used without further purification, while solvents were dried and purified by distillation, according to standard procedures. Petroleum ether refers to the fraction with a boiling point of $40-$ $60{ }^{\circ} \mathrm{C}$. The GAD was prepared and characterized according to the procedure reported in the ESI, and details about the synthesis, characterization, cytotoxicity and antioxidant properties are available in Sections S1-S6. $\dagger$

Collagen was purchased from Mascia Brunelli while thrombin was obtained from Merck Millipore (formerly SigmaAldrich).

\section{Platelet aggregation}

Blood collection and preparative procedures. Freshly drawn venous blood from healthy volunteers of the "Centro Trasfusionale, Ospedale San Martino" in Genoa was collected into a $130 \mathrm{mM}$ aqueous trisodium citrate anticoagulant solution $(9: 1)$. The donors claimed to have not taken drugs known to interfere with platelet function in the two weeks prior to blood collection and gave their informed consent, according to the ethical standards of the committee of our institution. In particular, under Italian legislation, it is not necessary to obtain the favorable opinion of the ethical committee in advance when using samples of blood provided from voluntary donors.

Washed platelets were prepared by centrifuging whole blood at $100 \times g$ for $20 \mathrm{~min}$.

The obtained platelet-rich plasma was then centrifuged at $1100 \times g$ for $15 \mathrm{~min}$.

The pellet was washed once with a pH 5.2 ACD solution (75 mM trisodium citrate, $42 \mathrm{mM}$ citric acid and $136 \mathrm{mM}$ glucose), centrifuged at $1100 \times g$ for $15 \mathrm{~min}$ and then re- suspended in pH 7.4 HEPES buffer (145 mM NaCl, $5 \mathrm{mM} \mathrm{KCl,}$ $1 \mathrm{mM} \mathrm{MgSO}, 10 \mathrm{mM}$ glucose, and $10 \mathrm{mM}$ HEPES).

\section{Compliance with ethics requirements (Italian legislation)}

The authors of this work declare that the samples analyzed in this study belong to the blood collection of "Centro Trasfusionale, Ospedale San Martino" in Genoa (Italy) and were stored in the Biochemistry Laboratory - Department of Pharmacy, Genoa University, according to the ethical standards of the committee of these institutions. Therefore, this study does not contain any experiments with human or animal subjects.

Determination of platelet aggregation inhibition. Platelet aggregation was performed in a Bio-Data aggregometer according to Born's method ${ }^{96}$ and quantified by light transmission reaching within $6 \mathrm{~min}$ at $37^{\circ} \mathrm{C}$. Briefly, washed platelets $\left(3.0 \times 10^{8}\right.$ plts per $\left.\mathrm{mL}\right)$ were preincubated with saline for $3 \mathrm{~min}$ at $37^{\circ} \mathrm{C}$ or GA or GAD solution before the addition of 0.1

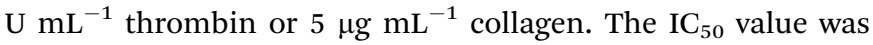
calculated as detailed above.

Intraplatelet ROS production. Intraplatelet ROS production was measured by flow cytometry using the properties of $2^{\prime}, 7^{\prime}$ dichlorodihydrofluorescein diacetate (DCFH-DA), which rapidly diffuses across cell membranes and is trapped within the cell following hydrolysis by cell esterase to $2^{\prime}, 7^{\prime}$-dichlorodihydrofluorescein (DCFH). DCFH is oxidized by ROS produced during platelet activation to form $2^{\prime}, 7^{\prime}$-dichlorofluorescein (DCF), which is a highly fluorescent molecule. ROS production was measured in washed platelets $\left(1.0 \times 10^{8}\right.$ $\mathrm{mL}^{-1}$ ) pre-incubated with saline, GA or the GAD for $15 \mathrm{~min}$ at $37{ }^{\circ} \mathrm{C}$ and then stimulated by $0.1 \mathrm{U} \mathrm{mL}^{-1}$ thrombin or $5 \mu \mathrm{g}$ $\mathrm{mL}^{-1}$ collagen. Incubation was stopped by putting the samples on ice, and then they were immediately analyzed in a Merck Millipore Bioscience Guava easyCyte flow cytometer to calculate the $\mathrm{IC}_{50}$ value. The percentage of inhibition, i.e. the extent of the inhibition of the maximal aggregation measured in the presence of the agent, was always compared with that measured in a control sample containing saline, carried out under the same conditions.

Statistical analysis for ROS and aggregation experiments. Reported data are the mean \pm SD obtained in at least six different experiments, each performed in duplicate. Student's $t$ test was performed for statistical analysis and $p<0.05$ was considered statistically significant.

\section{Bacterial strains}

A total of fourteen bacterial strains were evaluated in this study: eleven were chosen as representative of the Gram positive group while 3 belonged to the Gran-negative one. Of the total, eleven were of clinical origin, previously being isolated from different human specimens and identified according to standard procedures, ${ }^{97}$ while the others were from the American Type Culture Collection (ATCC).

Among the eleven Gram positive isolates, six belonged to the staphylococcal genus and were a methicillin resistant Staphylococcus aureus (MRSA), a methicillin susceptible $S$. aureus 
(MSSA), a methicillin resistant S. epidermidis (MRSE), and a methicillin susceptible $S$. epidermidis (MSSE) strain, as well as two reference strains S. aureus ATCC29813 (susceptible to methicillin) and S. epidermidis ATCC35984 (resistant to methicillin); four strains were from the Enterococcus genus and were E. faecalis resistant to glycopeptides (VRE), susceptible $E$. faecalis ATCC29212, E. faecium VRE and an E. faecium isolate susceptible to glycopeptides (VSE). A clinical strain of Micrococcus luteus was chosen as another Gram positive representative strain while single isolates of Escherichia coli, Klebsiella pneumoniae and Pseudomonas aeruginosa were chosen as Gram negative clinical strains.

\section{Susceptibility testing}

Antibacterial activities of free GA and the GAD were evaluated for selected bacterial strains. Minimal Inhibitory Concentrations (MICs) for the GAD and GA were determined following the microdilution method, detailed by the Clinical and Laboratory Standards Institute. ${ }^{85}$ Briefly, overnight cultures of bacteria were diluted to yield a final concentration of about $5 \times 105$ cells $\mathrm{mL}^{-1}$.

Samples were then added to equivalent volumes of various concentrations of GA and the GAD, previously appropriately diluted in dimethyl sulfoxide, distributed on a microplate and prepared from serial 2-fold dilutions, ranging from $256 \mu \mathrm{g} \mathrm{mL}^{-1}$ $(1506 \mu \mathrm{M})$ to $16 \mu \mathrm{g} \mathrm{mL} \mathrm{mL}^{-1}(94 \mu \mathrm{M})$ for GA and from $2048 \mu \mathrm{g} \mathrm{mL}^{-1}$ $(120 \mu \mathrm{M})$ to $128 \mu \mathrm{g} \mathrm{mL}^{-1}(7,5 \mu \mathrm{M})$ for the GAD, respectively.

After $24 \mathrm{~h}$ incubation at $37^{\circ} \mathrm{C}$, the lowest concentration of GA or the GAD that prevented visible growth was recorded as the MIC. All MICs were obtained in three independent sets of experiments and the most representative value was chosen as the MIC.

\section{Conclusions}

The GAD is a biodegradable, non-cytotoxic, nanosized dendrimer, peripherally esterified with sixty-four units of gallic acid (GA), and characterized by a spherical morphology. According to investigations, it is endowed with a strong antioxidant potency, higher than that of GA. It is also well performing as a preservative additive, able to prevent essential oil oxidative degradation with an efficiency far greater than that of GA. In the present work, the GAD was also assayed for evaluating its biological activity, i.e. its capability of inhibiting platelet aggregation, intra-platelet ROS production and multi-drug resistant bacterial growth. Platelet anti-aggregation and ROS inhibitory activities were investigated on two model systems, i.e. by using both thrombin and collagen as inducing agents. In both cases, the GAD was more efficient than GA by 7 times in inhibiting platelet aggregation and by 7-8 times in inhibiting ROS production. By a comparison with the literature, it was also found to be more active than aspirin and other natural polyphenols assayed under the same or similar conditions.

The antibacterial potency was evaluated against eleven Gram-positive strains of clinical relevance, including MRSA, MRSE and VRE.
The GAD is significantly more powerful than GA against Gram-positive species, by exerting activity at MIC $(\mu \mathrm{M})$ concentrations that were 11-50 times lower than those of GA. In addition, even if the literature data available are very poor and discordant, the GAD showed a higher activity in inhibiting the growth of the Gram-positive pathogens when compared to GA activities previously reported.

Based on these results, the GAD can be considered a semisynthetic, plant related nano-device, eligible as a therapeutic agent for the regulation of platelet aggregation and ROS production and therefore for the treatment of OS and related diseases. In addition the GAD is capable of fighting relevant bacterial infections including those for which common antibiotics fail.

\section{Conflicts of interest}

There are no conflicts to declare.

\section{Acknowledgements}

The authors would like to thank Mr G. Osvaldo for elemental analysis of the synthetic intermediates and the final compound during the new GAD preparation. Special thanks are due to Professor Deirdre Kantz, whose mother tongue is English, for correcting the language of the manuscript.

\section{References}

1 S. Di Meo, T. T. Reed, P. Venditti and V. M. Victor, Oxid. Med. Cell. Longevity, 2016, 2016, 1245049, DOI: 10.1155/2016/ 1245049.

2 B. Mayer and B. Hemmens, Trends Biochem. Sci., 1997, 22, 477-481.

3 T. P. A. Devasagayam, J. C. Tilak, K. K. Boloor, K. S. Sane, S. S. Ghaskadbi and R. D. Lele, J. Assoc. Physicians India, 2004, 52, 794-804.

4 D. G. Deavall, E. A. Martin, J. M. Horner and R. Roberts, J. Toxicol., 2012, 2012, 645460.

5 B. Badhani, N. Sharma and R. Kakkar, RSC Adv., 2015, 5, 27540-27557.

6 Z. M. Ruggeri, Nat. Med., 2002, 8, 1227-1234.

7 G. Davı, C. Patrono and N. Eng, J. Med., 2007, 357, 24822494.

8 P. M. Ridker, J. E. Buring and N. Rifai, Circulation, 2001, 103, 491-495.

9 G. Leoncini, D. Bruzzese, M. G. Signorello, U. Armani, A. Piana, D. Ghiglione and P. Camicione, Thromb. Haemostasis, 2007, 97, 218-227.

10 A. J. Lusis, Nature, 2000, 407, 233-241.

11 G. Leoncini, M. G. Signorello, A. Segantin, E. Giacobbe, U. Armani, A. Piana and P. Camicione, Thromb. Res., 2009, 124, e48-e55.

12 E. Medina and D. H. T. Pieper, Curr. Top. Microbiol. Immunol., 2016, 398, 3-33.

13 M. O. Ahmed and K. E. Baptiske, Microb. Drug Resist., 2018, 24, 590-606. 
14 R. Vivas, A. A. T. Barbosa, S. S. Dolabela and S. Jain, Microb. Drug Resist., 2019, 25, 890-908.

15 C. Carbon, Clin. Microbiol. Infect., 2000, 6(suppl. 2), 17-22.

16 L. B. Rice, Am. J. Infect. Control, 2006, 34, S11-S19, discussion S64-S73.

17 W. F. Oliveira, P. M. S. Silva, R. C. S. Silva, G. M. M. Silva, G. Machado, L. C. B. B. Coelho and M. T. S. Correia, J. Hosp. Infect., 2018, 98, 111-117.

18 D. J. Hetem, S. H. Rooijakkers and M. B. Ekkelenkamp, Staphylococci and Micrococci in Infectious Diseases, Elsevier, 4th edn, 2017, vol. 2, pp. 1509-1522.

19 I. Mack and J. Bielicki, Pediatr. Infect. Dis. J., 2019, 38, S33S38.

20 J. M. Gould, C. Gunasekera and A. Khan, Curr. Opin. Pharmacol., 2019, 48, 69-75.

21 K. W. Luo, J. G. Sun, J. Y. Chan, L. Yang, S. H. Wu, K. P. Fung and F. Y. Liu, Chemotherapy, 2011, 57, 449-459.

22 S. C. Renaud, R. Gueguen, J. Schenker and A. D'Houtaud, Epidemiology, 1998, 9, 184-188.

23 T. Hayek, B. Fuhrman, J. Vaya, M. Rosenblat, P. Belinky, R. Coleman, A. Elis and M. Aviramet, Arterioscler., Thromb., Vasc. Biol., 1997, 17, 2744-2752.

24 C. Cabrera, R. Artacho and R. Gimenez, J. Am. Coll. Nutr., 2006, 25, 79-99.

25 W. J. Kong, C. Jin, X. H. Xiao, Y. L. Zhao, Z. L. Li, P. Zhang, W. Liu and X. F. Li, J. Hazard. Mater., 2010, 179, 742-747.

26 J. M. Silvan, E. Mingo, M. Hidalgo, S. de Pascual-Teresa, A. V. Carrascosa and A. J. Martinez-Rodriguez, Food Control, 2013, 29, 25-31.

27 A. Khatkar, A. Nanda, P. Kumar and B. Narasimhan, Arabian J. Chem., 2017, 10, S2870-S2880.

28 G. A. Soto-Chilaca, N. Ramírez-Corona, E. Palou and A. López-Malo, Journal of Food Bioengineering and Nanoprocessing, 2016, 1, 165-181.

29 E. Obreque-Slier, A. Pena-Neira, R. Lopez-Solıs, F. ZamoraMarın, J. R. da Silva and O. Laureano, J. Agric. Food Chem., 2010, 58, 3591-3599.

30 M. Daglia, A. Di Lorenzo, S. F. Nabavi, Z. S. Talas and S. M. Nabavi, Curr. Pharm. Biotechnol., 2014, 15, 362-372.

31 L. A. de la Rosa, E. Alvarez-Parrilla and G. A. GonzalezAguilar, Fruit and Vegetable Phytochemicals: Chemistry, Nutritional Value and Stability, Wiley-Blackwell Hoboken, New Jersey (United States), 2016.

32 H. L. Madsen and G. Bertelsen, Trends Food Sci. Technol., 1995, 6, 271-277.

33 N. Nakatani, in Natural antioxidants from spices, ed. M. T. Huang, C. T. Ho and C. Y. Lee, ACS American Chemical Society, Washington, DC, 1st edn, 1992, vol. 6 pp. 72-86.

34 D. Li, Z. J. Liu, W. J. Zhao, Y. Xi and F. Niu, Process Biochem., 2011, 46, 2210-2214.

35 S. W. Kim, Y. W. Han, S. T. Lee, H. J. Jeong, S. H. Kim, I. H. Kim, S. O. Lee, D. G. Kim, S. H. Kim, S. Z. Kim and W. H. Park, Mol. Carcinog., 2008, 47, 114-125.

36 J. M. Kratz, C. R. Andrighetti-Fröhner, P. C. Leal, R. J. Nunes, R. A. Yunes, E. Trybala, T. Bergström, C. R. Barardi and C. M. Simões, Biol. Pharm. Bull., 2008, 31, 903-907.
37 M. S. Kang, J. S. Oh, I. C. Kang, S. J. Hong and C. H. Choi, J. Microbiol., 2008, 46, 744-750.

38 M. Kaur, B. Velmurugan, S. Rajamanickam, R. Agarwal and C. Agarwal, Pharm. Res., 2009, 26, 2133-2140.

39 K. C. Choi, Y. H. Lee, M. G. Jung, S. H. Kwon, M. J. Kim, W. J. Jun, J. Lee, J. M. Lee and H. G. Yoon, Mol. Cancer Res., 2009, 7, 2011-2021.

40 H. M. Chen, Y. C. Wu, Y. C. Chia, F. R. Chang, H. K. Hsu, Y. C. Hsieh, C. C. Chen and S. S. Yuan, Cancer Lett., 2009, 286, 161-171.

41 G. Sun, S. Zhang, Y. Xie, Z. Zhang and W. Zhao, Oncol. Lett., 2015, 11, 150-158.

42 K. Filipiak, M. Hidalgo, J. M. Silvan, B. Fabre, R. J. Carbajo, A. Pineda-Lucena, A. Ramos, B. de Pascual-Teresa and S. de Pascual-Teresa, Food Funct., 2014, 5, 381-389.

43 H. Sakagami and K. Satoh, Anticancer Res., 1997, 17, 221-224. 44 M. Inoue, R. Suzuki, N. Sakaguchi, Z. Li, T. Takeda, Y. Ogihara, B. Y. Jiang and Y. Chen, Biol. Pharm. Bull., 1995, 18, 1526-1530.

45 M. T. Mansouri, Y. Farbood, M. J. Sameri, A. Sarkaki, B. Naghizadeh and M. Rafeirad, Food Chem., 2013, 138, 1028-1033, DOI: 10.1016/j.foodchem.2012.11.022.

46 S. S. Chang, V. S. Lee, Y. L. Tseng, K. C. Chang, K. B. Chen, Y. L. Chen and C. Y. Li, J. Evidence-Based Complementary Altern. Med., 2012, 2012, 683872.

47 R. Diaz-Gomez, H. Toledo-Araya, R. Lopez-Solıs and E. Obreque-Slier, LWT-Food Sci. Technol., 2014, 59, 896-900.

48 A. Sarjit, Y. Wang and G. A. Dykes, Food Microbiol., 2015, 46, 227-233.

49 M. H. Liu, X. X. Wu, J. K. Li, L. Liu, R. G. Zhang, D. Y. Shao and X. D. Du, Food Control, 2017, 73, 613-618.

50 E. Sorrentino, M. Succi, L. Tipaldi, G. Pannella, L. Maiuro, M. Sturchio, R. Coppola and P. Tremonte, Int. J. Food Microbiol., 2018, 266, 183-189.

51 T. A. Vico, V. B. Arce, M. F. Fangio, L. B. Gende, C. A. Bertran, D. O. Mártire and M. S. Churio, J. Nanopart. Res., 2016, 18(348), 1-13.

52 S. Bhattacharyya, S. M. Ahammed, B. P. Saha and P. K. Mukherjee, AAPS PharmSciTech, 2013, 14, 1025-1033.

53 M. G. Ferruzzi, J. K. Lobo, E. M. Janle, N. Whittaker, B. Cooper, J. E. Simon, Q. L. Wu, C. Welch, L. Ho, C. Weaver and G. M. Pasinetti, J. Alzheimer's Dis., 2009, 18, 113-124.

54 Y. Konishi, Y. Hitomi and E. Yoshioka, J. Agric. Food Chem., 2004, 52, 2527-2532.

55 T. Yasuda, A. Inaba, M. Ohmori, T. Endo, S. Kubo and K. Ohsawa, J. Nat. Prod., 2000, 63, 1444-1446.

56 Z. Fang and B. Bhandaria, Trends Food Sci. Technol., 2010, 21, 510-523.

57 G. Cirillo, K. Kraemer, S. Fuessel, F. Puoci, M. Curcio, U. G. Spizzirri, I. Altimari and F. Iemma, Biomacromolecules, 2010, 11, 3309-3315.

58 Y. S. Cho, S. K. Kim, C. B. Ahn and J. Y. Je, Carbohydr. Polym., 1011, 83, 1617-1622.

59 G. Olga, C. Styliani and R. G. Ioannis, Food Chem., 2015, 185, 33-40. 
60 H. Hu, L. Nie, S. Feng and J. Suo, Pharmazie, 2013, 68, 401405.

61 Y. Deligiannakis, G. A. Sotiriou and S. E. Pratsinis, ACS Appl. Mater. Interfaces, 2012, 4, 6609-6617.

62 L. M. Kaminskas, B. J. Boyd and C. J. H. Porter, Nanomedicine, 2011, 6, 1063-1084.

63 C. C. Lee, J. A. MacKay, J. M. J. Frechet and F. C. Szoka, Nat. Biotechnol., 2005, 23, 1517-1526.

64 R. Hourani and A. Kakkar, Macromol. Rapid Commun., 2010, 31, 947-974.

65 S. Alfei, G. B. Taptue, S. Catena and A. Bisio, Chin. J. Polym. Sci., 2018, 36, 999-1010.

66 S. Alfei, S. Catena, M. Ponassi, C. Rosano, V. Zoppi and A. Spallarossa, Eur. J. Pharm. Sci., 2018, 124, 153-164.

67 S. Alfei, F. Turrini, S. Catena, P. Zunin, B. Parodi, G. Zuccari, A. M. Pittaluga and R. Boggia, New J. Chem., 2019, 43, 24382448.

68 X. Ma, Z. Zhou, E. Jin, Q. Sun, B. Zhang, J. Tang and Y. Shen, Macromolecules, 2013, 46, 37-42.

69 O. L. P. De Jesus, H. R. Ihre, L. Gagne, J. M. J. Frechet and F. C. Szoka, Bioconjugate Chem., 2002, 13, 453-461.

70 X. Ma, J. Tang, Y. Shen, M. Fan, H. Tang and M. Radosz, J. Am. Chem. Soc., 2009, 131, 14795-14803.

71 K. Jain, P. Kesharwani, U. Gupta and N. K. Jain, Int. J. Pharm., 2010, 394, 122-142.

72 S. Alfei, S. Catena and F. Turrini, Drug Delivery Transl. Res., 2019, ID: DDTR-D-19-00157, under review.

73 S. Alfei, P. Oliveri and C. Malegori, ChemistrySelect, 2019, 4, 8891-8901.

74 L. M. Kaminskas, B. J. Boyd and C. J. H. Porter, Nanomedicine, 2011, 6, 1063-1084.

75 C. C. Griffin, J. Soc. Cosmet. Chem., 1954, 5, 249-256.

76 H. S. Chaudhari, R. R. Popat, V. S. Adhao and V. N. Shrikhande, J. Appl. Pharm. Res., 2016, 4, 1-19.

77 K. Manjappa and J. Narayanaswamy, Biomacromolecules, 2009, 42, 7353-7359.

78 A. Poole, J. M. Gibbins, M. Turner, M. J. van Vugt, J. G. van de Winkel, T. Saito, V. L. Tybulewicz and S. P. Watson, EMBO J., 1997, 16, 2333-2341.

79 D. E. Roberts, A. McNicol and R. Bose, J. Biol. Chem., 2004, 279, 19421-19430.

80 S. R. Coughlin, Thromb. Haemostasis, 1999, 82, 353-356.
81 A. Petroni, M. Blasevich, M. Salami, N. Papini, G. F. Montedorol and C. Galli, Thromb. Res., 1995, 78, 151160.

82 H. S. Lee, Bioresour. Technol., 2006, 97, 1372-1376.

83 K. Kim, O.-N. Bae, K.-M. Lim, J.-Y. Noh, S. Kang, K. Y. Chung and J.-H. Chung, J. Pharmacol. Exp. Ther., 2012, 343, 704-711.

84 J. Qiaoa, J. F. Arthurd, E. E. Gardinere, R. K. Andrewsd, L. Zenga and K. Xua, Redox Biol., 2018, 14, 126-130.

85 N. Carrim, J. F. Arthur, J. R. Hamilton, E. E. Gardiner, R. K. Andrews, N. Moran, M. C. Berndt and P. Metharom, Redox Biol., 2015, 6, 640-647.

86 Clinical and Laboratory Standards Institute, Performance standards for antimicrobial susceptibility testing. Sixteenth informational supplement. M100-S16, CLSI, Wayne, PA, 2006.

87 R. Pacheco-Ordaz, A. Wall-Medrano, M. G. Goni, G. RamosClamont-Montfort, J. F. Ayala-Zavala and G. A. GonzalezAguilar, Lett. Appl. Microbiol., 2017, 66, 25-31.

88 N. Rattanata, S. Klaynongsruang, C. Leelayuwat, T. Limpaiboon, A. Lulitanond, P. Boonsiri, S. Chio-Srichan, S. Soontaranon, S. Rugmai and J. Daduang, Int. J. Nanomed., 2016, 11, 3347-3356.

89 K. Zare, H. Nazemyeh, F. Lotfipour, S. Farabi, M. Ghiamirad and A. Barzegari, Pharm. Sci., 2014, 20, 6-11, http://pharmsci.tbzmed.ac.ir.

90 A. Borges, C. Ferreira, M. J. Saavedra and M. Simões, Microb. Drug Resist., 2013, 19, 256-265, DOI: 10.1089/mdr.2012.0244.

91 S. H. M. Al-Zahrani, J. Am. Sci., 2012, 8, 7-12.

92 L. Fu, W. Q. Lu and X. M. Zhou, BioMed Res. Int., 2016, 2016, 4287461, DOI: 10.1155/2016/4287461.

93 M. J. Alves, I. C. F. R. Ferreira, H. J. C. Froufe, R. M. V. Abreu, A. Martins and M. Pintado, J. Appl. Microbiol., 2013, 115, 346-357.

94 J. Gutiérrez-Fernández, M. R. García-Armesto, R. ÁlvarezAlonso, P. del Valle, D. de Arriaga and J. Rúa, J. Dairy Sci., 2013, 96, 4912-4920, DOI: 10.3168/jds.2013-6643.

95 V. Kuete, in Fighting Multidrug Resistance with Herbal Extracts, Essential Oils and Their Components, ed. M. Rai and K. Kon, Elsevier, USA, 2013, vol. 3, pp. 23-30.

96 G. V. R. Born, Nature, 1962, 194, 927-929, http:// www.ncbi.nlm.nih.gov/pubmed/13871375.

97 P. R. Murray, E. J. Baron, M. A. Pfaller, F. C. Tenover and R. H. Yolken, Manual of Clinical Microbiology, ASM Press, Washington, DC, 7th edn, 1999. 